\section{Gene silencing as a threat to the success of gene therapy}

\author{
Timothy H. Bestor
}

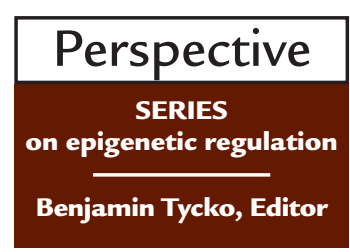

Department of Genetics and Development, College of Physicians and Surgeons of Columbia University, 701 West 168th Street, New York, New York 10032, USA. Phone: (212) 305-5331; Fax: (212) 740-0992; E-mail: THB12@columbia.edu.

Eukaryotes deploy elaborate defensive systems to protect the structure of their genomes and to oppose the expression of abnormal or alien transcription units. In mammals, the insertion of retroviral DNA or the incorporation of repeat arrays can trigger transcriptional silencing of the inserted sequences, usually via mechanisms that involve methylation of DNA within regulatory regions. Fungi and plants have similar methylation-based systems and also employ diverse post-transcriptional mechanisms to reduce or eliminate the expression of alien transcription units, mechanisms that operate even in organisms that apparently lack the capacity to methylate their DNA. Given their ubiquity in other eukaryotes, it is likely that similar post-transcriptional silencing mechanisms will be found to operate in mammals as well. I argue here that gene silencing mechanisms are diverse and efficient and are likely to represent a barrier to many forms of gene therapy. For this reason, it will be necessary to develop gene therapy vectors that are both resistant to host defensive measures and efficient in terms of delivery and expression.

Gene therapy usually depends on a construct or recombinant virus that directs expression of an agent (protein or RNA) in a particular tissue. Delivery to the target tissue has long been recognized as a difficult problem, as has proper cell type-specific regulation. The existence of gene silencing, the recognition and inactivation of alien genes by target cells (reviewed in ref. 1), has only been recently recognized as an additional challenge to gene therapy. Many cases are known in which a transferred gene undergoes a brief period of expression followed by a decline to undetectable levels without the loss of the expression construct. Gene silencing in mammals is likely to be more common than currently believed, although the responsible mechanisms are diverse and still rather poorly understood. Some of the more prominent forms of gene silencing that are likely to compromise gene therapy studies are briefly described in this review.

Retrovirus silencing. Most gene silencing is the result of host defenses against transposable elements and retroviruses (2), and silencing may be especially troublesome in the case of retroviral delivery vectors because of the strong host response that such agents provoke. It has long been known that endogenous retroviruses are generally not expressed despite intact promoters in their long terminal repeats (LTRs), and that retroviral genomes introduced by infection of preimplantation mouse embryos usually are transmitted intact to progeny but are silent (3). The latter effect has prevented the use of retroviral vectors in the construction of transgenic mouse strains. The fact that microinjected DNA constructs give more predictable expression in transgenic animals than does infection with recombinant retroviruses demonstrates that cells have the ability to recognize and inactivate retroviral DNA (4).

The mechanism of long-term transcriptional silencing of retroviral DNA involves methylation of the 5 position of cytosines present within promoter elements. DNA methylation inhibits transcription largely by the recruitment of repressive chromosomal proteins such as histone deacetylases (reviewed in ref. 5). Early data had shown that retroviral LTR sequences are normally densely methylated, and that de novo methylation on newly integrated retroviral DNA is much more efficient in embryonic stem (ES) cells than in differentiated somatic cells (6). Jaenisch and colleagues (7) showed that retroviruses in somatic tissues could be reactivated by injection of 5-azacytidine, an irreversible inhibitor of DNA methyltransferases. This finding suggests that cytosine methylation rather than position effects or other chromatin-dependent effects is responsible for retrovirus silencing. It has been suggested that the cell recognizes structural features diagnostic of retroviral integration intermediates and that this recognition event promotes de novo methylation of local sequences (4).

Palmer et al. (8) found that, whereas cultured fibroblasts infected with recombinant retroviruses supported long-term expression of an adenosine deaminase (ADA) transgene, transplantation of these cells into the skin of rats caused a drop in ADA expression of more than 1,000-fold within 30 days. Extinction in this case did not involve loss of infected cells, and treatments with the DNA methyltransferase inhibitor 5-azacytidine or the histone deacetylase inhibitor sodium butyrate had only small effects 
on ADA activity. The authors could not identify the cause of silencing, but later work showed that use of an internal Fibronectin promoter between the retroviral LTRs permitted prolonged expression after engraftment of infected cells, suggesting that the silencing effect is directed against the LTR (9). Halene et al. (10) modified the LTR of a retroviral vector that is normally subject to strong silencing in hematopoietic and lymphoid cells and reported increased resistance to silencing. Long-term expression-on the order of years, rather than weeks-does not seem to have been demonstrated for any retroviral vector. Resistance to silencing must be an important consideration in the design of retroviral vectors for purposes of gene therapy.

Repeat-induced gene silencing. Selker (reviewed in ref. 11) noted that sequence duplications induced methylation of both copies during the sexual cycle of the fungus Neurospora crassa; many $\mathrm{C} \rightarrow \mathrm{T}$ transition mutations also appeared within the duplicated sequences (the active mutation process thus far appears to be unique to $N$. crassa). Matzke and Matzke methylation and silencing of sequence duplications in tobacco plants (reviewed in ref. 12). Later it was found that transgene copy number was inversely proportional to the level of expression in some lines of transgenic mice (13), and Garrick et al. (14) showed that a repeat array upstream of a reporter gene in transgenic mice caused methylation and silencing of the transgene; removal of the array via Cre-mediated excision relieved the silencing and reduced methylation of the transgene promoter. This experiment proved that sequence duplications can cause silencing of linked promoters in transgenic mice. The effect is variable; some transgenes at some locations are not subject to copy number effects, whereas others are very sensitive (reviewed in ref. 12).

Repeat-induced gene silencing is associated with methylation in most cases, but multicopy transgenes are also subject to silencing in Drosophila melanogaster, which has no detectable modified bases in its DNA (15). In plants and mammals, it appears that long-term transcriptional silencing of multicopy transgenes depends on cytosine methylation, although methylation-independent mechanisms may exist as well. Repeat-induced gene silencing has clear relevance for any gene therapy approach that is likely to lead to the insertion of multicopy arrays.

The efficiency of silencing via the interaction of repeated sequences depends on the nature of the construct, number of repeats, and site of integration in a manner that is not yet possible to predict. DNA sequence features of the vector or transgene may also influence the duration of transgene expression. Dinucleotide frequencies in the human genome differ markedly from those of other organisms; in particular, human DNA has low frequencies of $\mathrm{CPG}$ dinucleotides except in regions of very high $\mathrm{G} / \mathrm{C}$ contents (the $\mathrm{CpG}$ islands that are associated with many genes). Other genomes, particularly those of bacteria, tend to be relatively rich in $\mathrm{CpG}$ dinucleotides, even in regions of moderate G/C content. Scrable and Stambrook (16) showed that a lacI gene of Escherichia coli was inactivated by methylation in transgenic mice, but a synthetic version of the gene that followed human codon usage but encoded the same polypeptide was relatively resistant to methylation and silencing. This result suggests that regions of DNA whose dinucleotide frequencies do not coincide with those of the host genome can elicit a silencing response.

Post-transcriptional silencing. Gene silencing can also occur at the RNA level even while transcription proceeds at high rates. First noted as cosuppression or sense suppression in plants, and more recently as post-transcriptional gene silencing (PTGS), expression of high levels of a transgene-encoded RNA can cause drastic reductions of both homologous endogenous transcripts and of the transgene-encoded transcript (reviewed in ref. 16). The effect also removes viral RNA and is thought to protect the organism from infection by exogenous viruses and from the activation of endogenous transposons. Hamilton and Baulcombe (17) showed that a small antisense RNA of about 25 nucleotides accumulates in plant tissue that is undergoing PTGS and that this antisense RNA might provide target specificity to this RNA degradation process. It is thought that the antisense RNA is produced by an RNA-dependent RNA polymerase, and that contact between sense and antisense RNAs activates specific RNA degradation. The effect is transmissible to adjacent plant tissue, which thereby gains resistance to specific viruses before exposure to the virus (reviewed in ref. 16).

A phenomenon called RNA interference (RNAi) has fundamental similarities to PTGS and depends on short duplex or foldback RNAs, which trigger degradation of homologous RNA (18). RNAi was first observed in Caenorhabditis elegans but has been reported to occur in a wide variety of metazoa, including fruit flies and zebra fish (18 and references therein). Substoichiometric amounts of double-stranded RNA lead to the degradation of much larger amounts of target RNA. Injection of double stranded RNA homologous to a cellular gene into C. elegans produces a phenotype that is in many cases indistinguishable from a null mutation in that gene. The recent finding of Wianny and Zernicka-Goetz that double-stranded RNA can be used to inhibit expression of specific genes in mouse embryos provides the first direct evidence for RNAi in mammalian cells (19). Given the ubiquity of PTGS in all other major taxa, it thus appears very likely that similar mechanisms will be found to interfere with the expression of foreign genes humans and that this may complicate gene therapy efforts.

In addition to purely post-transcriptional effects, aberrant RNAs can feed back to cause transcriptional silencing and methylation of homologous DNA sequences. The effect has to date been confirmed only in plants (reviewed in ref. 5). The aberrant RNA can be chimeric for different sequences, which can lead to the silencing of endogenous genes homologous to 1 com- 
ponent of the chimeric RNA, or can contain inverted repeats or other sequence arrangements that differ from those of the target gene (reviewed in ref. 5). The mechanism is unknown, but is likely to involve direct interactions of the gene and aberrant RNA. It should be noted that the mechanisms involved in virtually every form of gene silencing remain to be discovered, and research in this area is likely to accelerate in the near future.

Conclusion. Many gene silencing effects (especially post-transcriptional effects) were first observed in plants or fungi, and some have not yet been reported from studies of mammals. Does this mean that they are weaker or nonexistent in mammals? Not necessarily, as mammalian transcription is still widely regarded as an elaboration of the model of the lac operon derived by Jacob and Monod, and departures from this model do not always receive objective consideration. It is striking that post-transcriptional gene silencing was observed in a wide range of taxa before its documentation in a mammal, despite the fact that the overwhelming majority of gene expression studies are conducted on mammals. However, it is now clear that mammalian genes can be inactive or silent, even in the presence of all the factors normally sufficient for their expression, and that cells can detect alterations of their genomes and respond by imposing a strong and heritable silencing effect. It has been noted that host-mediated genesilencing pathways could be used to extinguish the expression of harmful genes (20); for example, silencing of the $B C R$ promoter in BCR-ABL1-positive cases of chronic myelogenous leukemia would be very likely to contribute to remission of the disease, and silencing of the CCR5 gene would be expected to confer resistance to infection by HIV-1. This approach has many of the virtues of a vaccine in the sense that an existing host defense system is stimulated; other approaches to specific downregulation do not enjoy this advantage (20). On the other hand, gene silencing has already compromised a number of gene transfer efforts (for examples, see refs. 9 and 21) and is likely to represent a barrier to many of the forms of gene therapy currently under development. Even if the delivery and regulation problems can be solved, it is not unlikely that successful gene transfer and tissue-specific expression may be followed by dwindling expression and loss of therapeutic effect unless silencing-resistant expression constructs are developed and used.

\section{Acknowledgments}

I apologize to those authors whole work could not be directly cited due to length limitations. I am grateful to M. Matzke, E. Selker, and B. Tycko for discussions, and to J. Ryan for comments on the manuscript. Supported by grants from the National Institutes of Health (GM59377 and HD37687) and by a grant from the Leukemia Society of America.

1. Bestor, T.H., Chandler, V.L., and Feinberg, A.P. 1994. Epigenetic effects in eukaryotic gene expression. Dev. Genet. 15:458-462.

2. Yoder, J.A., Walsh, C.P., and Bestor, T.H. 1997. Cytosine methylation and the ecology of intragenomic parasites. Trends Genet. 13:335-340.

3. Berleth, T., Nobis, P., Jaenisch, R., and Harbers, K. 1987. Activation of endogenous retroviral genomes in Mov strains of mice. J. Gen. Virol. 68:2919-2923.

4. Bestor, T.H., and Tycko, B. 1996. Creation of genomic methylation patterns. Nat. Genet. 12:363-367.

5. Wolffe, A.P., and Matzke, M.A. 1999. Epigenetics: regulation through repression. Science. 286:481-486.

6. Lei, H., et al. 1996. De novo DNA cytosine methyltransferase activities in mouse embryonic stem cells. Development. 122:3195-3205.

7. Jaenisch, R., Schnieke, A., and Harbers, K. 1986. Treatment of mice with 5 -azacytidine efficiently activates silent retroviral genomes in different tissues. Proc. Natl. Acad. Sci. USA. 82:1451-1455.

8. Palmer, T.D., Rosman, G.J., Osborne, W.R.A., and Miller, A.D. 1991. Genetically modified skin fibroblasts persist long after transplantation but gradually inactivate introduced genes. Proc. Natl. Acad. Sci. USA. 88:1330-1334.

9. Wei, M.Q., et al. 1999. Sustained gene expression in transplanted skin fibroblasts in rats. Gene Ther. 6:840-844.

10. Halene, S., et al. 1999. Improved expression in hematopoietic and lymphoid cells in mice after transplantation of bone marrow transduced with a modified retroviral vector. Blood. 94:3349-3357.

11. Selker, E.U. 1997. Epigenetic phenomena in filamentous fungi: useful paradigms or repeat-induced confusion? Trends Genet. 13:296-301.

12. Matzke, A.J., and Matzke, M.A. 1998. Position effects and epigenetic silencing of plant transgenes. Curr. Opin. Plant Biol. 1:142-148.

13. Mehtali, M., LeMeur, M., and Lathe, R. 1990. The methylation-free status of a housekeeping transgene is lost at high copy number. Gene. 91:179-184.

14. Garrick, D., Fiering, S., Martin, D.I., and Whitelaw, E. 1998. Repeatinduced gene silencing in mammals. Nat. Genet. 18:56-59.

15. Dorer., D.R., and Henikoff, S. 1997. Transgene repeat arrays interact with distant heterochromatin and cause silencing in cis and trans. Genetics. 147:1181-1190.

16. Scrable, H., and Stambrook, P.J. 1997. Activation of the lac repressor in the transgenic mouse. Genetics. 147:297-304.

17. Hamilton, A.J., and Baulcombe, D.C. 1999. A species of small antisense RNA in postranscriptional gene silencing in plants. Science. 286:950-952.

18. Tuschl, T., Zamore, P.A., Lehmann, R., Bartel, D.P., and Sharp, P.A. 1999. Targeted mRNA degradation by double stranded RNA in vitro. Genes Dev. 13:3191-3197.

19. Wianny, F., and Zernicka-Goetz, M. 2000. Specific interference with gene function by double-stranded RNA in early mouse development. Nat. Cell Biol. 2:70-75.

20. Xu, G.-L., and Bestor, T.H. 1997. Cytosine methylation targeted to pre-determined sequences. Nat. Genet. 17:376-378.

21. Harvey, B.G., et al. 1999. Airway epithelial CFTR mRNA expression in cystic fibrosis patients after repetitive administration of a recombinant adenovirus. J. Clin. Invest. 104:1245-1255. 